\title{
Wavelet-based analysis of the regular and chaotic dynamics of rectangular flexible plates subjected to shear-harmonic loading
}

\author{
J. Awrejcewicz ${ }^{\mathrm{a}, \mathrm{b}, *}$, I.V. Papkova ${ }^{\mathrm{c}}$, E.U. Krylova ${ }^{\mathrm{c}}$ and V.A. Krysko ${ }^{\mathrm{c}}$ \\ ${ }^{\mathrm{a}}$ TU Darmstadt LOEWE-Zentrum AdRIA, Darmstadt, Germany \\ ${ }^{\mathrm{b}}$ TU Lodz, Department of Automation and Biomechanics, Lodz, Poland \\ ${ }^{\mathrm{c}}$ Department of Mathematics and Modeling, The Saratov State Technical University, Saratov, Russia
}

\begin{abstract}
We investigate non-linear dynamics of flexible rectangular plates subjected to external shear harmonic load action. We show that an application of the classical and widely used Fourier analysis does not allow to obtain real picture of the frequency vibration characteristics in each time instant. On the other hand, we show that application of the wavelets approach allows to follow frequency time evolutions. Our numerical results indicate that vibrations in different plate points occur with the same frequencies set although their power is different. Hence, the vibration characteristics can be represented by one arbitrary taken plate point. Furthermore, using wavelets scenarios of transitions from regular to chaotic dynamics are illustrated and discussed including two novel scenarios not reported so far in the existing literature.
\end{abstract}

Keywords: Dynamics, plate, chaos, wavelets

\section{Introduction}

Plates are widely applied in various branches of industry like aviations, ship construction, civil engineering etc. Since the plate-members of the constructions are in general subjected to action of various loading actions, they need careful modeling and validated numerical investigations. Today's industrial needs are mainly focused on the parameters choice of the analyzed construction to keep them working in safe regimes. However, in particular chaotic dynamics of structures is not enough studied so far. Continual systems as plates have been analyzed in references [1,2]. Although chaotic dynamics has been detected in fluid mechanics relatively long time ago, both bifurcation and chaotic dynamics exhibited by plates and shells have been studied just recently [3-12]. Our contribution is aimed on a study of chaotic vibrations of flexible rectangular plates subjected to shear loading harmonic action, which belongs to novel and challenging open problems.

Mathematical models of flexible rectangular plates and shells have been constructed using the kinematic model of Kirchhoff-Love with inclusion of non-linear relations between deformations and displacements. This leads to a system of non-homogeneous partial nonlinear differential equations regarding stresses and strains. In order to reduce the continuous system to a lumped one may use the Finite Difference Method (FDM) with approximation $O\left(h^{2}\right)$, which allows for investigation of the studied rectangular plates as the mechanical objects with infinite degrees of freedom. The obtained system of ODEs is then solved via the fourth order Runge-Kutta method.

\footnotetext{
*Corresponding author: J. Awrejcewicz, TU Darmstadt LOEWE-Zentrum AdRIA, Bartningstr. 53, 64289 Darmstadt, Germany and TU Lodz, Department of Automation and Biomechanics, 1/15 Stefanowskiego St., 90-924 Lodz, Poland. E-mail: awrejcew@p.lodz.pl.
} 


\section{Governing equations}

We consider a plate with a constant rigidity and density within a classical non-linear theory subjected to action of harmonic shear loading. In the initial time interval $t \in[0 ; 1]$ a small static transversal load is applied. In a 3D co-ordinates the investigated plate can be represented in the following form: $\Omega=\left\{x_{1}, x_{2}, x_{3} \mid\left(x_{1}, x_{2}\right) \in[0 ; a] \times[0 ; b]\right.$, $\left.x_{3} \in[-h ; h]\right\}, 0 \leqslant t<\infty$.

We study the following non-dimensional PDEs governing dynamics of the shallow shells:

$$
\begin{aligned}
& \frac{1}{12\left(1-\mu^{2}\right)}\left(\nabla_{\lambda}^{4} w\right)-L(w, F)+\frac{\partial^{2} w}{\partial t^{2}}+\varepsilon \frac{\partial w}{\partial t}-q\left(x_{1}, x_{2}, t\right)+2 S \frac{\partial^{2} w}{\partial x \partial y}=0, \\
& \nabla_{\lambda}^{4} F+\frac{1}{2} L(w, w)=0
\end{aligned}
$$

where $\nabla_{\lambda}^{4}=\frac{1}{\lambda^{2}} \frac{\partial^{4}}{\partial x_{1}^{4}}+\lambda^{2} \frac{\partial^{4}}{\partial x_{2}^{4}}+2 \frac{\partial^{4}}{\partial x_{1}^{2} \partial x_{2}^{2}}, L(w, F)=\frac{\partial^{2} w}{\partial x_{1}^{2}} \frac{\partial^{2} F}{\partial x_{2}^{2}}+\frac{\partial^{2} w}{\partial x_{2}^{2}} \frac{\partial^{2} F}{\partial x_{1}^{2}}-2 \frac{\partial^{2} w}{\partial x_{1} \partial x_{2}} \frac{\partial^{2} F}{\partial x_{1} \partial x_{2}}$ is known non-linear operator, $w$ and $F$ are functions of deflection and stress, respectively.

System (Eq. (1)) is reduced to a non-dimensional form using the following non-dimensional parameters: $\lambda=a / b ; x_{1}=a \bar{x}_{1}, x_{2}=a \bar{x}_{2}, w=2 h \bar{w}$ denotes deflection; $F=E(2 h)^{3} \bar{F}$ is the stress function; $t=t_{0} \bar{t}$ denotes time; $q=\frac{E(2 h)^{4}}{a^{2} b^{2}} \bar{q}$ is the external loading; $\varepsilon=(2 h) \bar{\varepsilon}$ is the damping coefficient of a surrounding medium, and $S=\frac{E(2 h)^{3}}{a b} \bar{S}$ is the external shear loading. Bars over the non-dimensional parameters are omitted. Furthermore, the following notations are introduced; $a, b$ denote plate length regarding $x_{1}$ and $x_{2}$, respectively, and $\mu$ is the Poisson coefficient.

The following boundary conditions are attached to Eq. (1):

1. Support on flexible non-stretched (non-compressed) ribs

$$
\begin{array}{ll}
w=0, & \frac{\partial^{2} w}{\partial x_{1}^{2}}=0, \quad F=0, \quad \frac{\partial^{2} F}{\partial x_{1}^{2}}=0 \quad \text { for } x_{1}=0,1 \\
w=0, & \frac{\partial^{2} w}{\partial x_{2}^{2}}=0, \quad F=0, \quad \frac{\partial^{2} F}{\partial x_{2}^{2}}=0 \quad \text { for } x_{2}=0,1 .
\end{array}
$$

2. Clamping on a contour

$$
\begin{array}{ll}
w=0, & \frac{\partial w}{\partial x_{1}}=0, \quad F=0, \quad \frac{\partial F}{\partial x_{1}}=0 \quad \text { for } x_{1}=0,1 \\
w=0, & \frac{\partial w}{\partial x_{2}}=0, \quad F=0, \quad \frac{\partial F}{\partial x_{2}}=0 \quad \text { for } x_{2}=0,1 .
\end{array}
$$

3. Free support on a contour

$$
\begin{array}{ll}
w=0, & \frac{\partial^{2} w}{\partial x_{1}^{2}}=0, \quad F=0, \quad \frac{\partial F}{\partial x_{1}}=0 \quad \text { for } x_{1}=0,1 \\
w=0, & \frac{\partial^{2} w}{\partial x_{2}^{2}}=0, \quad F=0, \quad \frac{\partial F}{\partial x_{2}}=0 \quad \text { for } x_{2}=0,1
\end{array}
$$


4. Clamping with flexible non-stretched (non-compressed) ribs

$$
\begin{aligned}
& w=0, \quad \frac{\partial w}{\partial x_{1}}=0, \quad F=0, \quad \frac{\partial^{2} F}{\partial x_{1}^{2}}=0 \quad \text { for } x_{1}=0,1, \\
& w=0, \quad \frac{\partial w}{\partial x_{2}}=0, \quad F=0, \quad \frac{\partial^{2} F}{\partial x_{2}^{2}}=0 \quad \text { for } x_{2}=0,1
\end{aligned}
$$

The following initial conditions are applied

$$
\left.w\left(x_{1}, x_{2}\right)\right|_{t=0}=0, \frac{\partial w}{\partial t}=0
$$

\section{Finite Difference Method (FDM)}

In what follows we consider the Finite Difference Method with approximation $O\left(h^{2}\right)$ regarding spatial co-ordinates $x_{1}$ and $x_{2}$. In this case an application of the FDM to the infinite system of degrees-of-freedom governed by PDEs (Eq. (1)) yields a finite lumped system governed by the following difference-operator equations

$$
\begin{aligned}
& -\frac{1}{12\left(1-\mu^{2}\right)}\left(\lambda^{-2} \Lambda_{1}^{2} w_{i j}+2 \Lambda_{12}^{2} w_{i j}+\lambda^{2} \Lambda_{2}^{2} w_{i j}\right)-\Lambda_{1} w_{i j} \cdot \Lambda_{2} F_{i j}-\Lambda_{2} w_{i j} \cdot \Lambda_{1} F_{i j}+ \\
& +\Lambda_{12} w_{i j} \cdot \Lambda_{12} F_{i j}+q_{i}-P_{x} \Lambda_{2}-P_{y} \Lambda_{1}-2 S \Lambda_{12}=\left(w_{t t}+\varepsilon w_{t}\right)_{i, j} \\
& \left(\lambda^{-2} \Lambda_{1}^{2} F_{i j}+2 \Lambda_{12}^{2} F_{i j}+\lambda^{2} \Lambda_{2}^{2} F_{i j}\right)=-\Lambda_{1} w_{i j} \cdot \Lambda_{2} w_{i j}+\left(\Lambda_{12} w_{i j}\right)^{2}, \\
& \text { where } \Lambda_{i} y=\frac{1}{h_{i}^{2}}\left[y\left(x_{i}-h_{i}\right)-2 \cdot y\left(x_{i}\right)+y\left(x_{i}+h_{i}\right)\right], i=1,2 \\
& \Lambda_{12} y=\frac{1}{4 h_{1} h_{2}}\left[y\left(x_{1}+h_{1}, x_{2}+h_{2}\right)+y\left(x_{1}-h_{1}, x_{2}-h_{2}\right)-\left(x_{1}+h_{1}, x_{2}-h_{2}\right)-\left(x_{1}-h_{1}, x_{2}+h_{2}\right)\right], \\
& \Lambda_{i}^{2} y=\frac{1}{h_{i}^{4}}\left[y\left(x_{i}-2 h_{i}\right)-4 y\left(x_{i}-h_{i}\right)+6 y\left(x_{i}\right)-4 y\left(x_{i}+h_{i}\right)+y\left(x_{i}+2 h_{i}\right)\right], i=1,2 \\
& \Lambda_{12}^{2} y=\frac{1}{h_{1}^{2} h_{2}^{2}}\left[y\left(x_{1}-h_{1}, x_{2}-h_{2}\right)-2 y\left(x_{1}-h_{1}, x_{2}\right)+y\left(x_{1}-h_{1}, x_{2}-h_{2}\right)-2\left(x_{1}, x_{2}-h_{2}\right)+\right. \\
& \left.4 y\left(x_{1}, x_{2}\right)-2 y\left(x_{1}, x_{2}+h_{2}\right)+y\left(x_{1}+h_{1}, x_{2}-h_{2}\right)-2\left(x_{1}+h_{1}, x_{2}\right)+y\left(x_{1}+h_{1}, x_{2}+h_{2}\right)\right]
\end{aligned}
$$

The following initial conditions are attached to system (Eq. (7)):

$$
w_{i j}=f_{1}\left(x_{1 k}, x_{2 k}\right), w_{n}^{\prime}=f_{2}\left(x_{1 k}, x_{2 k}\right),(0 \leqslant k \leqslant n), 0 \leqslant t<\infty,
$$

whereas the boundary value conditions (Eqs $(2-5)$ ) are formulated in the following form:

1. Free support on a contour:

$$
\begin{aligned}
& w_{n, j}=0, \quad w_{n, j}=-w_{n-2, j}, \quad F_{n, j}=0, \quad F_{n, j}=-F_{n-2, j}, \quad j=1, \ldots, m-1, \\
& w_{i, m}=0, \quad w_{i, m}=-w_{i, m-2}, \quad F_{i, m}=0, \quad F_{i, m}=-F_{i, m-2}, \quad i=\overline{1, n-1} ;
\end{aligned}
$$


2. Clamping on a contour:

$$
\begin{aligned}
& w_{n, j}=0, \quad w_{n, j}=w_{n-2, j}, \quad F_{n, j}=0, \quad F_{n, j}=F_{n-2, j}, \quad j=1, \ldots, m-1, \\
& w_{i, m}=0, \quad w_{i, m}=w_{i, m-2}, \quad F_{i, m}=0, \quad F_{i, m}=F_{i, m-2}, \quad i=1, \ldots, n-1 \text {; }
\end{aligned}
$$

3. Support on flexible non-stretched (non-compressed) ribs:

$$
\begin{aligned}
& w_{n, j}=0, \quad w_{n, j}=-w_{n-2, j}, \quad F_{n, j}=0, \quad F_{n, j}=F_{n-2, j}, \quad j=1, \ldots, m-1, \\
& w_{i, m}=0, \quad w_{i, m}=-w_{i, m-2}, \quad F_{i, m}=0, \quad F_{i, m}=F_{i, m-2}, \quad i=1, \ldots, n-1 ;
\end{aligned}
$$

4. Clamping with flexible non-stretched (non-compressed) ribs:

$$
\begin{aligned}
& w_{n, j}=0, \quad w_{n, j}=w_{n-2, j}, \quad F_{n, j}=0, \quad F_{n, j}=-F_{n-2, j}, \quad j=1, \ldots, m-1, \\
& w_{i, m}=0, \quad w_{i, m}=w_{i, m-2}, \quad F_{i, m}=0, \quad F_{i, m}=-F_{i, m-2}, \quad i=1, \ldots, n-1 ;
\end{aligned}
$$

System of Eeqs (7-11) is solved via the fourth order Runge-Kutta method, where on each time step a large system of algebraic linear equations is solved. The Runge principle allows to choose a time step properly.

\section{Analysis and results}

We consider a rectangular plate with homogeneous boundary conditions (Eq. (8)) and the initial conditions $f_{1}\left(x_{1 k}, x_{2 k}\right)=0, f_{2}\left(x_{1 k}, x_{2 k}\right)=0$ being subjected to action of a shear-harmonic load in the form of $S=s_{0} \sin \omega_{p} t$, where $\omega_{p}$ and $s_{O}$ is the frequency and amplitude of the external excitations, respectively. Damping coefficient $\varepsilon=1$, whereas the Poisson's coefficient $\mu=0.3$.

We investigate numerically a convergence of the FDM versus a number $n$ of partition of intervals $[0 ; 1]$ and $[0 ; 1]$ of the rectangular plate in a regular (periodic) and chaotic regimes.

Let us study first the point $A\left(s_{01}, \omega_{1}\right)=A(8.6,13.4) \in\left\{s_{0}, \omega_{p}\right\}$ belonging to a periodic zone. Three curves for $n=m=12,14,16$ are reported in Fig. 1. Increasing $n$ causes initially decrease of the output signals amplitude, whereas for $n=m=14,16$ the curves fully overlap.

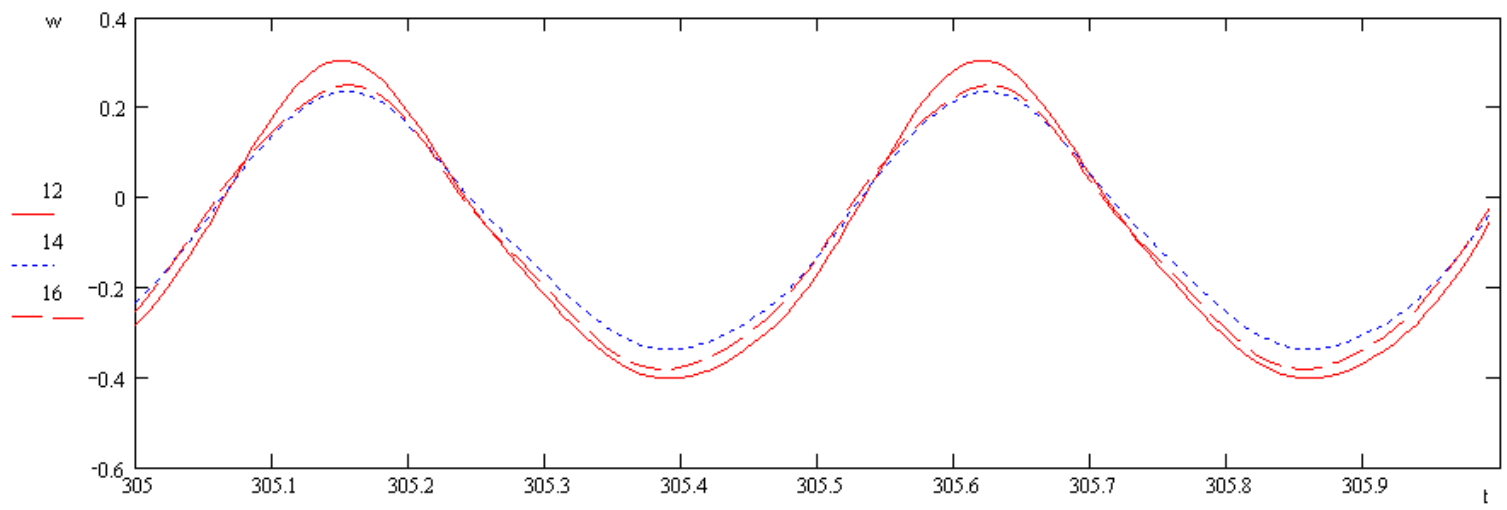

Fig. 1. Deflection $w(t)$ versus $n$ in a periodic zone.

Let us consider one more point $B\left(s_{02}, \omega_{1}\right)=B(28.7,13.4) \in\left\{s_{0}, \omega\right\}$, but in a chaotic zone. For all $n=12,14,16$ the studied signals differ from each other (see Fig. 2). 


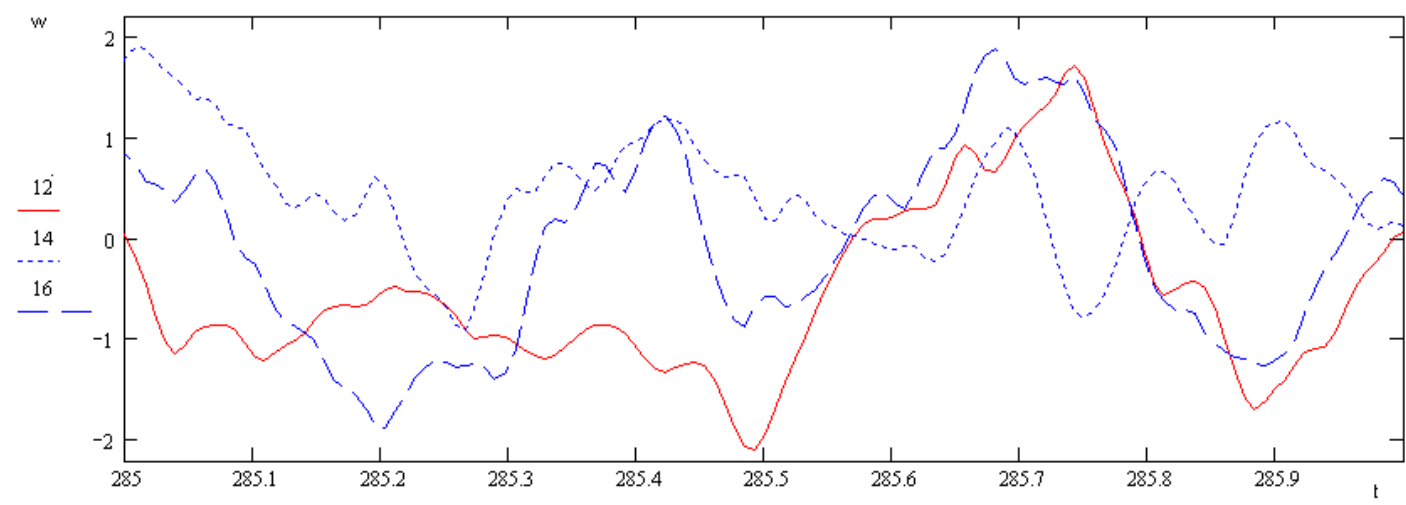

Fig. 2. Deflection $w(t)$ versus $n$ in a chaotic zone.

In what follows we study convergence of the FDM with respect to computation of the Lyapunov exponents. For this purpose we monitor the Lyapunov exponents evolution with time $(280 \leqslant t \leqslant 285)$ for a number of partitions $(n=12,14,16)$ in periodic and chaotic zones.

In point $A\left(s_{01}, \omega_{1}\right)=A(8.6,13.4) \in\left\{s_{0}, \omega_{p}\right\}$ the Lyapunov exponents for all studied partitions are negative, which indicates that in the investigated point $\left(A\left(s_{01}, \omega_{1}\right)=A(8.6,13.4) \in\left\{s_{0}, \omega_{p}\right\}\right)$ vibrations are periodic (Fig. 3).

On the other hand, in the point $B\left(s_{02}, \omega_{1}\right)=B(28.7,13.4) \in\left\{s_{0}, \omega\right\}$ the Lyapunov exponents for all partition numbers are positive, which proves occurrence of chaos in that point (Fig. 3).

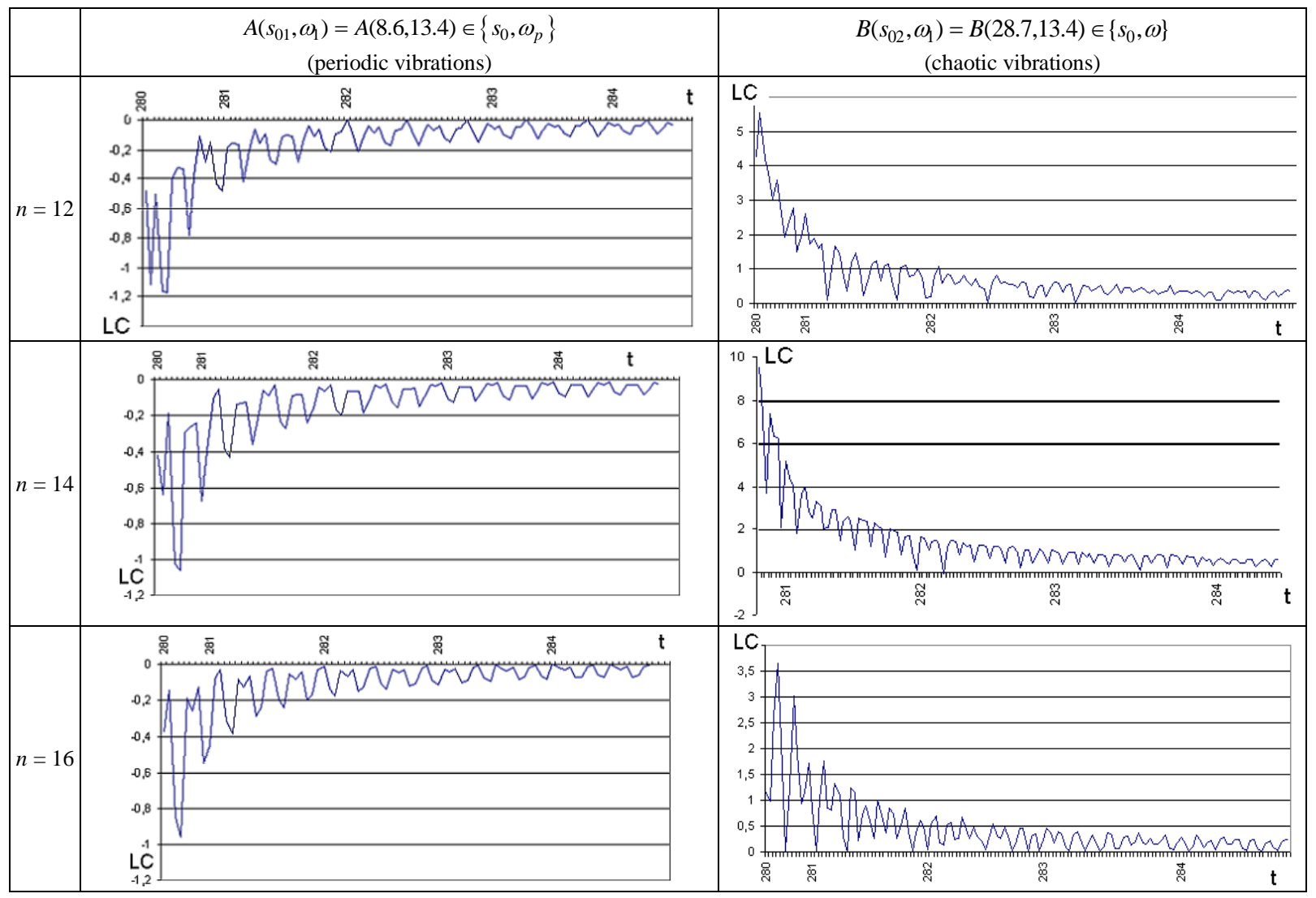

Fig. 3. The Lyapunov exponents time histories versus $n$ in periodic and chaotic zones. 
Let us construct a wavelet spectrum for the points $A\left(s_{01}, \omega_{1}\right)=A(8.6,13.4) \in\left\{s_{0}, \omega\right\}$ and $B\left(s_{02}, \omega_{1}\right)=$ $B(28.7,13.4) \in\left\{s_{0}, \omega\right\}$ for different numbers of partition $(n=12,14,16)$ in the governing Eqs $(7-8)$. The obtained results are reported in Table 1 for point $A$ and for point $B$ in Table 2.

Table 1

Convergence of the FDM in a periodic zone

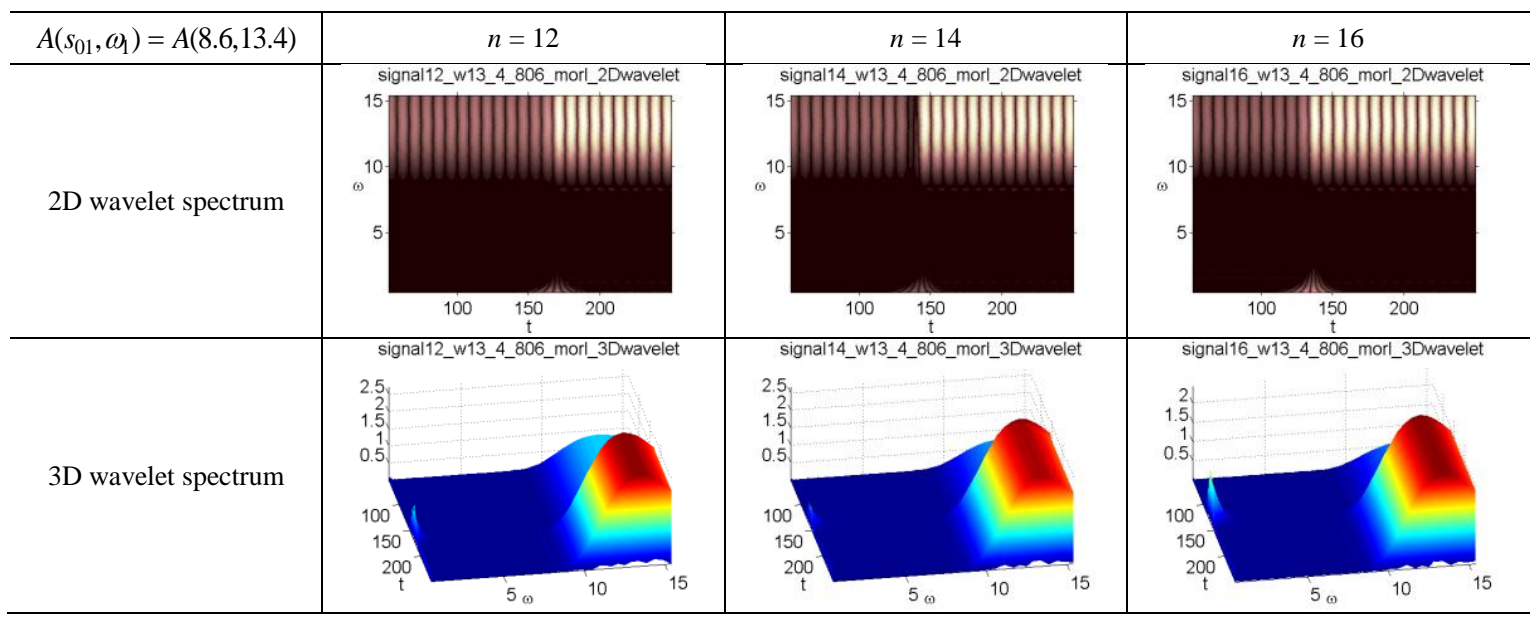

Table 2

Convergence of the FDM in a chaotic zone

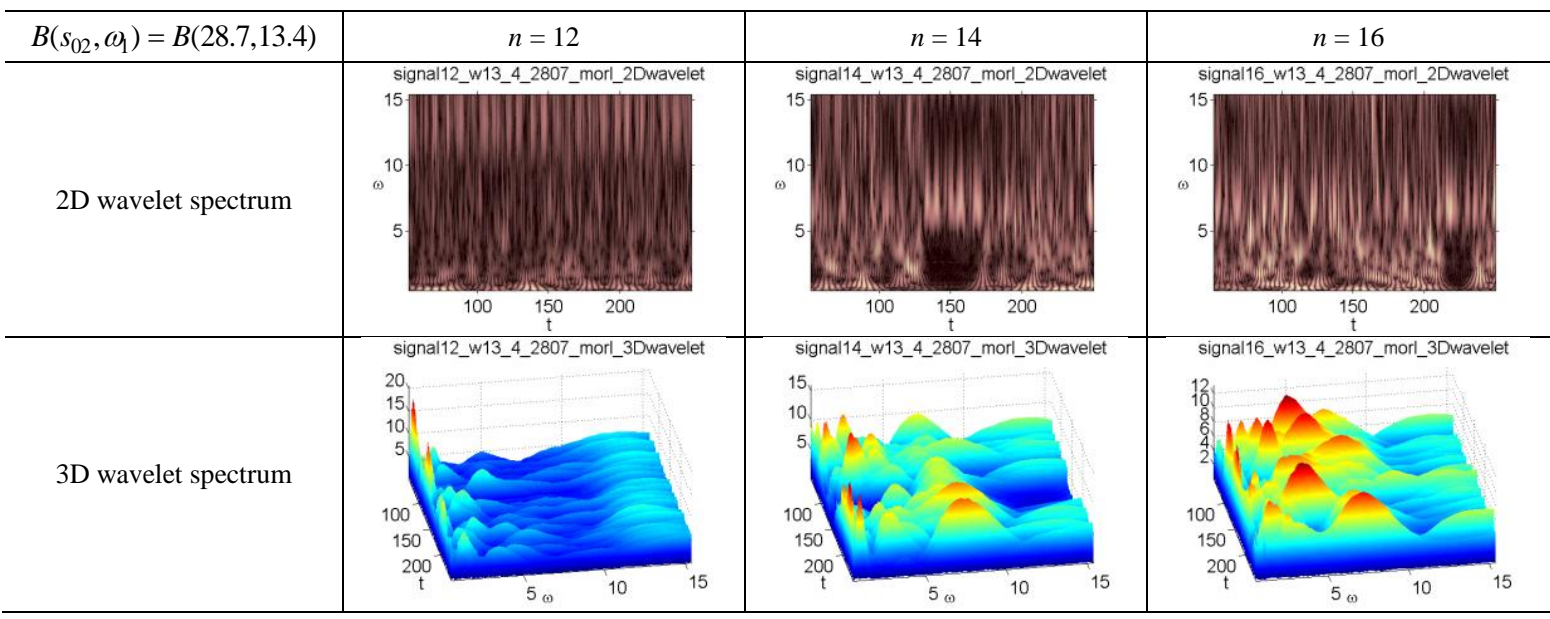

Numerical experiment shows that the wavelet spectrum does not depend on the partition numbers in the periodic zone. In the chaotic zone also reasonable good coincidence of the wavelet spectra is observed for different $n$. However, increase of power of the frequencies associated with chaos occurrence is observed. In other words it is clearly evident that the applied wavelets analysis can be viewed as a mathematical microscope. Namely, increase of partition numbers improves a power of our microscope. The so far illustrated and discussed results allow to formulate a conclusion that the applied FDM is convergent regarding both wavelet spectrum and the Lyapunov exponents.

It should be emphasized that although an increase of $n$ in the applied FDM improves essentially the obtained results, but it requires exponential increase of the computational time (one needs to solve a system of algebraic equations on each computational step, i.e. for $n=m=8$ we have 64 equations, whereas for $n=m=14$ we have 256 equations). 
Recall that the carried out so far investigations regarding the applied FDM allow to conclude that the numerical convergence is achieved in the average sense, i.e. with regard to the wavelet spectrum and Lyapunov exponents. In a chaotic zone the convergence with respect to the time histories comparison is not achieved. However, in the latter case an integral convergence, i.e. with respect to the wavelet spectrum is achieved.

Here we address another problem: is it reasonable to study only one plate point and then how to share the obtained knowledge to all plate points? In order to clarify this point we study five arbitrary taken points of the middle plate surface with the following co-ordinates $A(3 ; 3), B(n-2 ; 3), C(n-2 ; n-2), D(3 ; n-2)$, and $S(n / 2 ; n / 2)$ being a central middle surface point (Fig. 4).

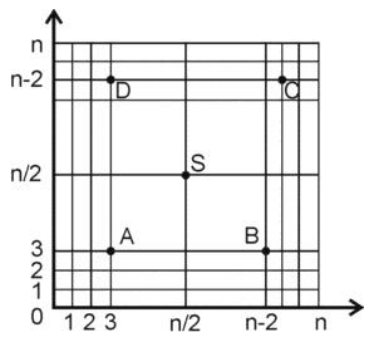

Fig. 4. Positions of points A, B, C, D, S on the middle plate surface.

Analysis of each of the mentioned points is supplemented by monitoring of evolution of time-frequency characteristics of the wavelet spectra with increase of ot the external load amplitude. The following external excitation frequencies have been taken into account: $\omega_{\mathrm{p}}=2.9, \omega_{\mathrm{p}}=5.8$ (this corresponds to natural plate vibration frequency), $\omega_{\mathrm{p}}=8.7$, and $\omega_{\mathrm{p}}=26, \omega_{\mathrm{p}}=13.4$. Some of the obtained results are reported in Tables 3 -5 . Since the frequency of compressing load possess essentially higher power in comparison to remaining frequencies, therefore the remaining frequencies are often not visible in the wavelet spectrum owing to their small magnitudes. Therefore, our results are reported for frequencies in a neighborhood of the excitation frequency.

Yet, it has been shown numerically that vibrations in different plate points are realized through the same frequency spectrum, although the frequencies power may differ in different points. In addition, the frequencies may vanish or again appear in time, but the spectrum record remains conserved. Consequently, it has been shown that a choice of only one plate vibration is sufficient for the plate vibrations investigation. We take, following tradition, its center as the representative one point.

One of the fundamental tasks of the investigation relies on monitoring a transition from regular to chaotic vibrations of our rectangular plate subjected to periodic excitation action. It should be emphasized that the classical approach based on the Fourier spectrum analysis is not appropriate to detect and analyze the mentioned scenarios.

Observe that during a study of the plate vibrations via the Fourier analysis a few disagreements in comparison to other tested characteristics may appear. Namely, in contrary to the frequency power spectrum indicating a harmonic behavior other characteristics show the quasi-periodic one. In Table 6 the following signal characteristics are reported: a time history $w(0.5 ; 0.5 t)$ for $280 \leqslant t \leqslant 286$, a phase portrait $w\left(w_{t}^{\prime}\right)$, a frequency power spectrum $S\left(\omega_{p}\right)$ and a Poincare map $w_{t}\left(w_{t+T}\right)$ for the center of the middle plate surface subjected to action of the load $S=s_{0} \sin \omega_{p} t$ with the frequency $\omega_{\mathrm{p}}=5.8$ (it is equal to plate natural vibration frequency) and with the amplitude $\mathrm{s}_{0}$ $=13.4$.

Application of the wavelet transformation have proved that the observed differences of the signals are not accidental. In contrary, a wavelet spectrum illustrates the fact that a transition from regularity to chaos is realized via frequencies, whose power is so small that it cannot be detected using the standard Fourier analysis. In the case of excitation frequency $\omega_{\mathrm{p}}=5.8$ a scenario to chaos has been obtained via a successive period doubling bifurcation. Our wavelet analysis contradicts this statement, because the first bifurcation occurs long earlier than that reported in the Fourier spectrum (Fig. 5). Figure 5 shows the first bifurcation for $\omega_{1}=2.9$, as well as the low frequency components. 


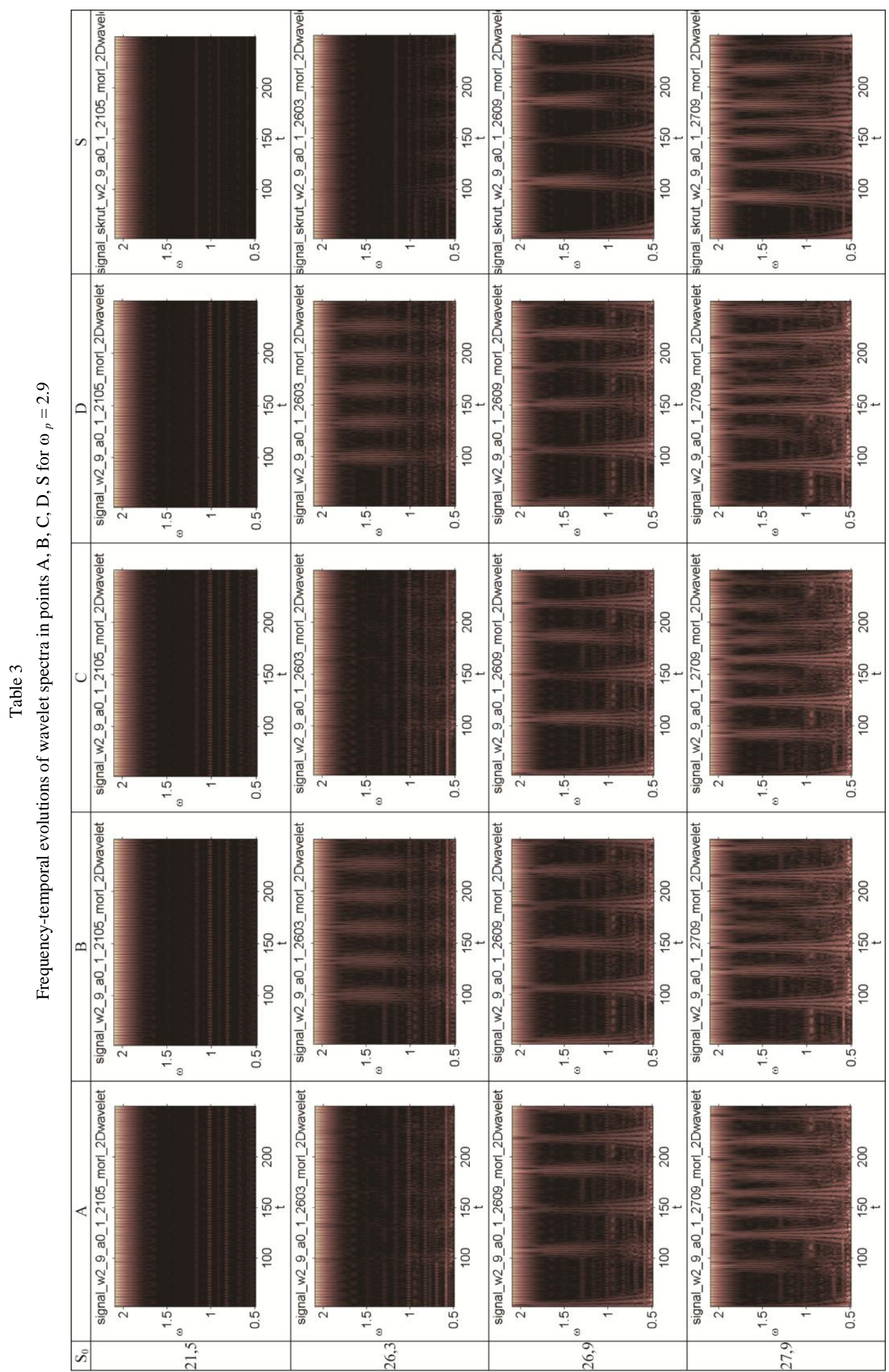




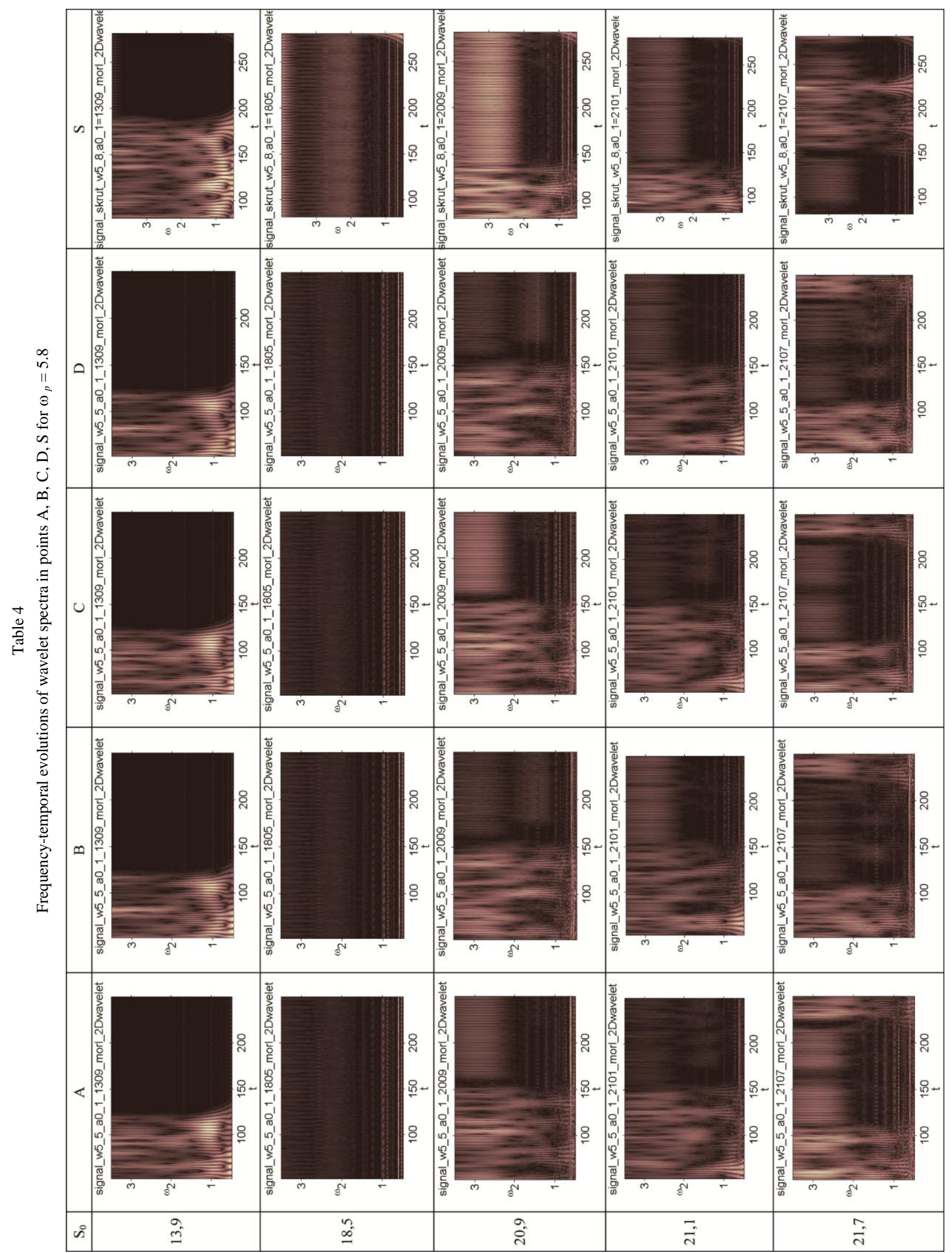




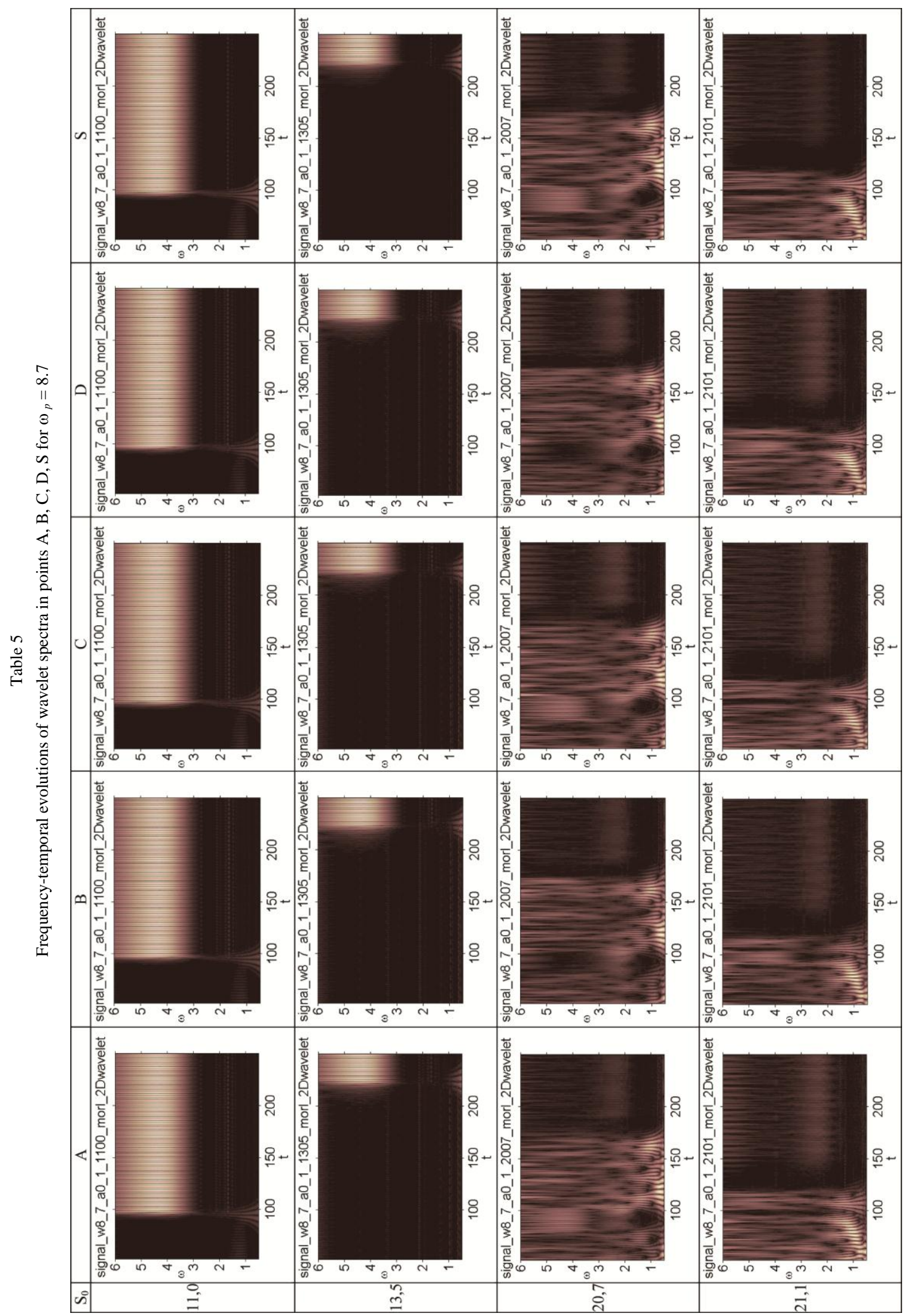


Table 6

Dynamical characteristics $\left(\omega_{\mathrm{p}}=5.8, \mathrm{~s}_{0}=13.4\right)$

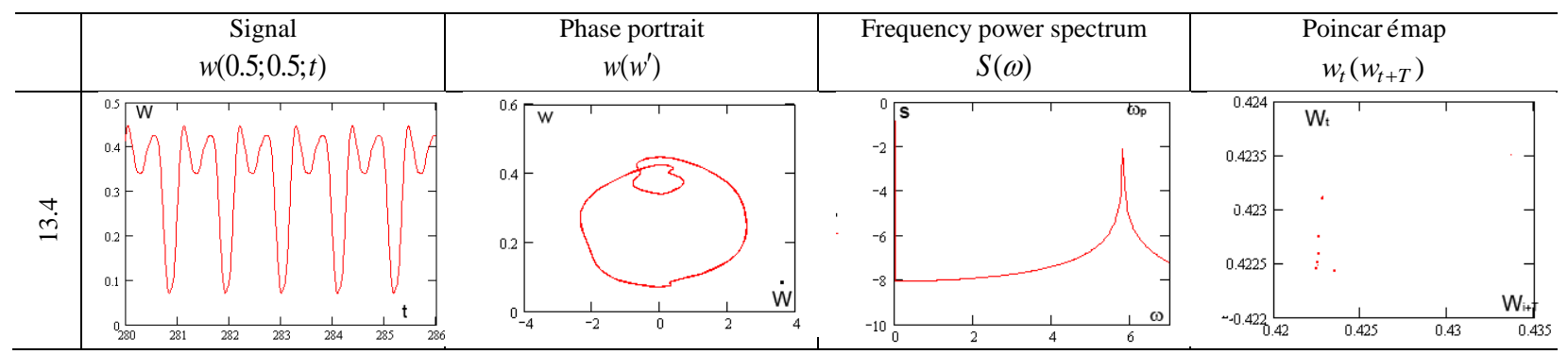

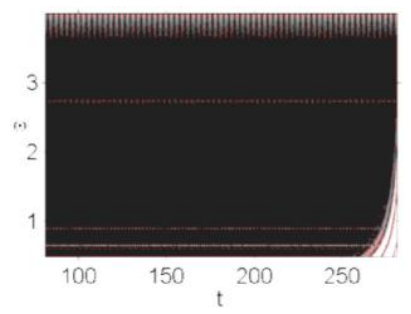

Fig. 5. Wavelet spectrum corresponding to the data of Table 6.

It should be noted that during our numerical investigations with $\omega_{\mathrm{p}}=2.9$ and $\omega_{\mathrm{p}}=8.7$ chaotic behavior has not been detected via the classical Fourier analysis. For $\omega_{\mathrm{p}}=8.7$ and with a small amplitude of excitation the obtained Fourier spectrum has been noisy and hence it was not possible to get conclusion regarding plate vibrations type (Table 7). In the case of $\omega_{p}=2.9$, increasing the external load amplitude up to 21.2 (frequency power spectrum has indicated periodic vibrations although other dynamic characteristics have not validated this observation).

However, using wavelet analysis it is possible to understand and explain the occurred situation. Namely, a transition from regular to chaotic dynamics for $\omega_{\mathrm{p}}=8.7$ has been realized via intermittency. The Fourier transformation does not allow to trace evolution of the frequency characteristics in time, since it produces a sum of all frequencies appearing in the whole investigated time interval. It is evident that an intermittency occurrence even on a short time interval makes the spectrum noisy and practically a scenario from regular to chaotic dynamics cannot be detected.

We are aimed on detection of scenario transitions from regular to chaotic vibrations of the rectangular plate subjected to shear-periodic load action. Let us monitor this transition for $\omega_{\mathrm{p}}=8.7$ (Table 7). For small values of the excitation amplitude $\left(\mathrm{s}_{0}=9.6\right)$ plate vibrations are sub-harmonic with the frequency $\left(\omega_{1}=4.35\right)$, and the latter frequency cancels the excitation frequency or equivalently a synchronization between two frequencies takes place.

For $\mathrm{s}_{0}=11.2$ a qualitative change of the plate vibrations in time is visible. In the initial time interval a low frequency component dominates together with the excitation frequency, whereas for any $t$ from interval $(100 ; 250)$ the plate vibration character is identical to that of $\mathrm{s}_{0}=9.6$.

Further external excitation produces in time $\omega_{1}=4.35\left(\mathrm{~s}_{0}=13.5\right)$. The power of excitation frequency increases, it becomes dominating one, and a low frequency component occurs in time interval $(150 ; 250)\left(\mathrm{s}_{0}=18.7\right)$. However, frequency $\omega_{1}$ does not vanish, but is rather shifted and its contribution to the power spectrum is negligible. We may observe it again in time interval $52 \leqslant t \leqslant 200\left(\omega_{1}=4.0\right)$ in Fig. 6, where also low frequency components are reported.

Further increase of the excitation amplitude is associated with occurrence of the intermittency islands for $t \geqslant 150\left(\mathrm{~s}_{0}=18.8\right)$. Increasing our control parameter yields a birth of frequency $\omega_{1}=4.35$.

For $\mathrm{s}_{0}=20.2$ and $t \leqslant 125$ the investigated plate exhibits chaotic dynamics, which with increase of the excitation amplitude fills up the whole wavelet spectrum. On the other hand, a 3D wavelet spectrum proves that chaos occurs on low frequencies.

We have illustrated and discussed how our investigated plate is transited into its chaotic state through the successive doubled frequency transformation into half of the excitation frequency. 


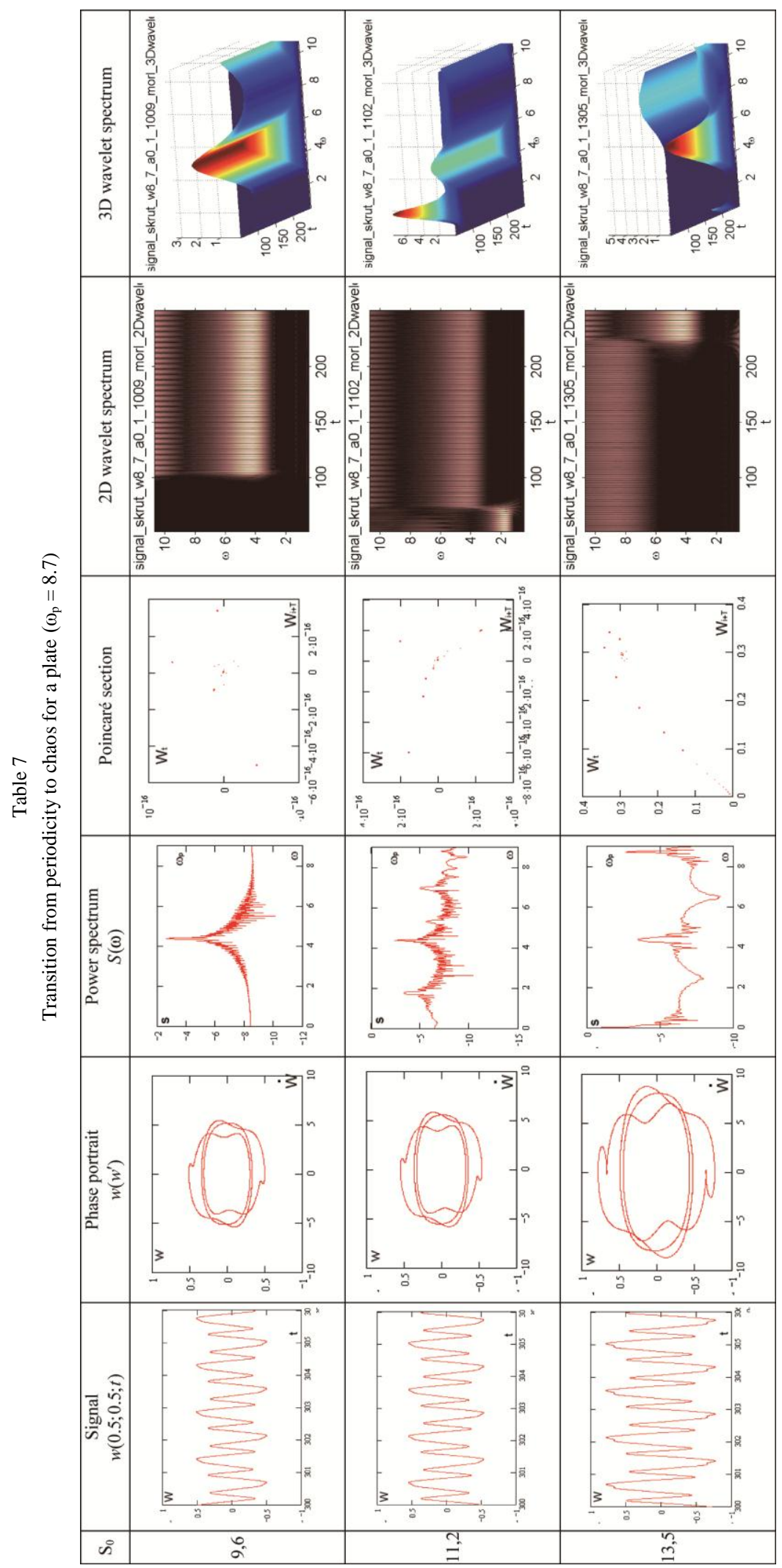




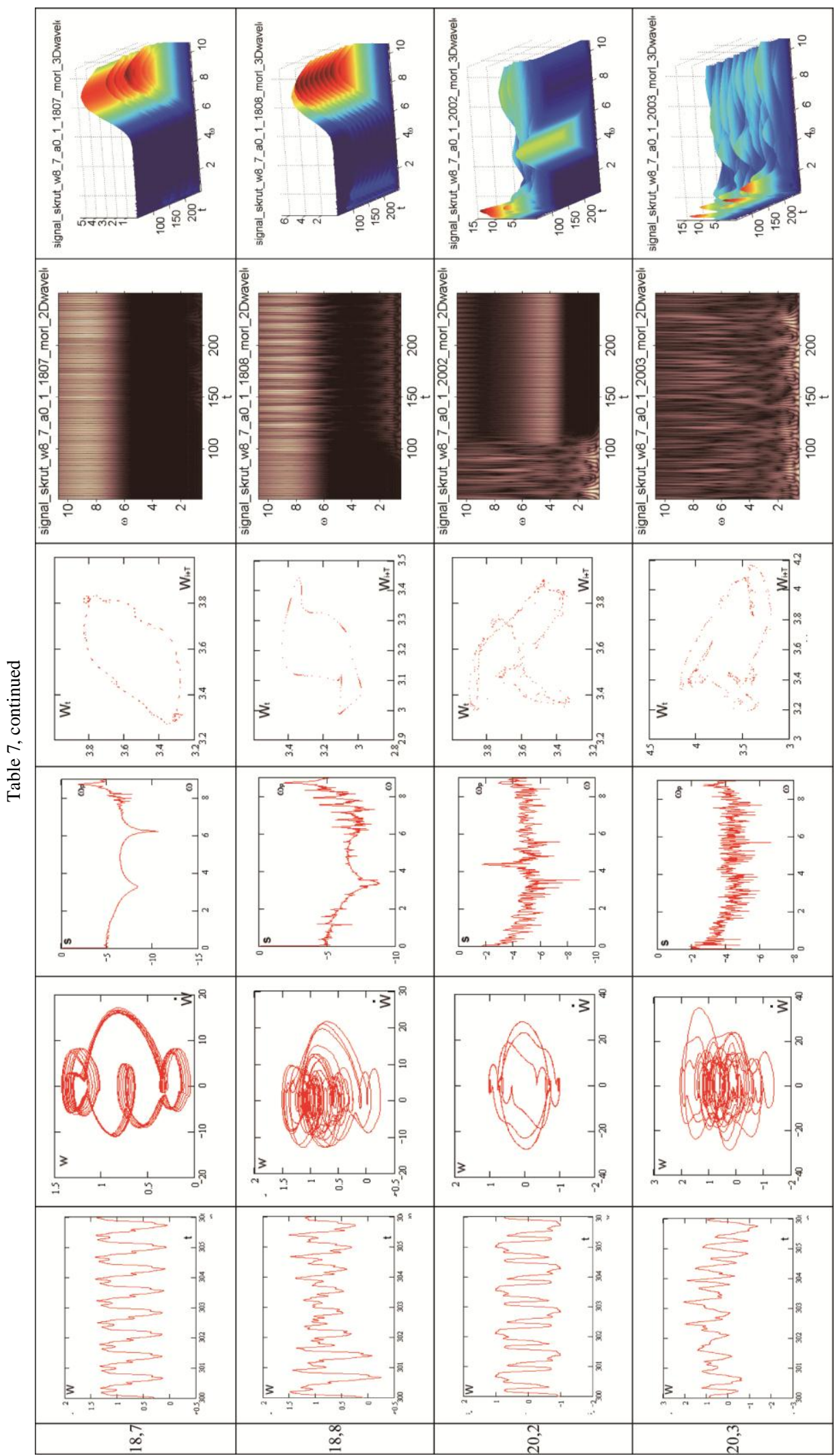




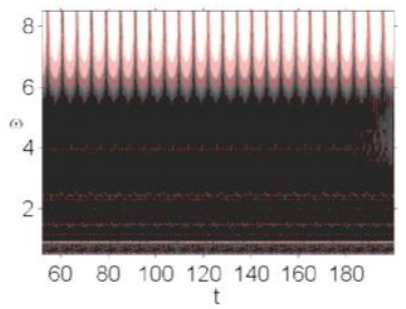

Fig. 6. Wavelet spectrum in interval of $52 \leqslant \mathrm{t} \leqslant 200, \mathrm{~s}_{0}=18.7, \omega_{\mathrm{p}}=8.7$.

In the case of external frequency $\omega_{p}=2.9$ a scenario yielding plate chaotic state is reported in Table 8 , which we briefly discuss below.

Table 8

Transition from periodicity to chaos for a plate $\left(\omega_{\mathrm{p}}=2.9\right)$

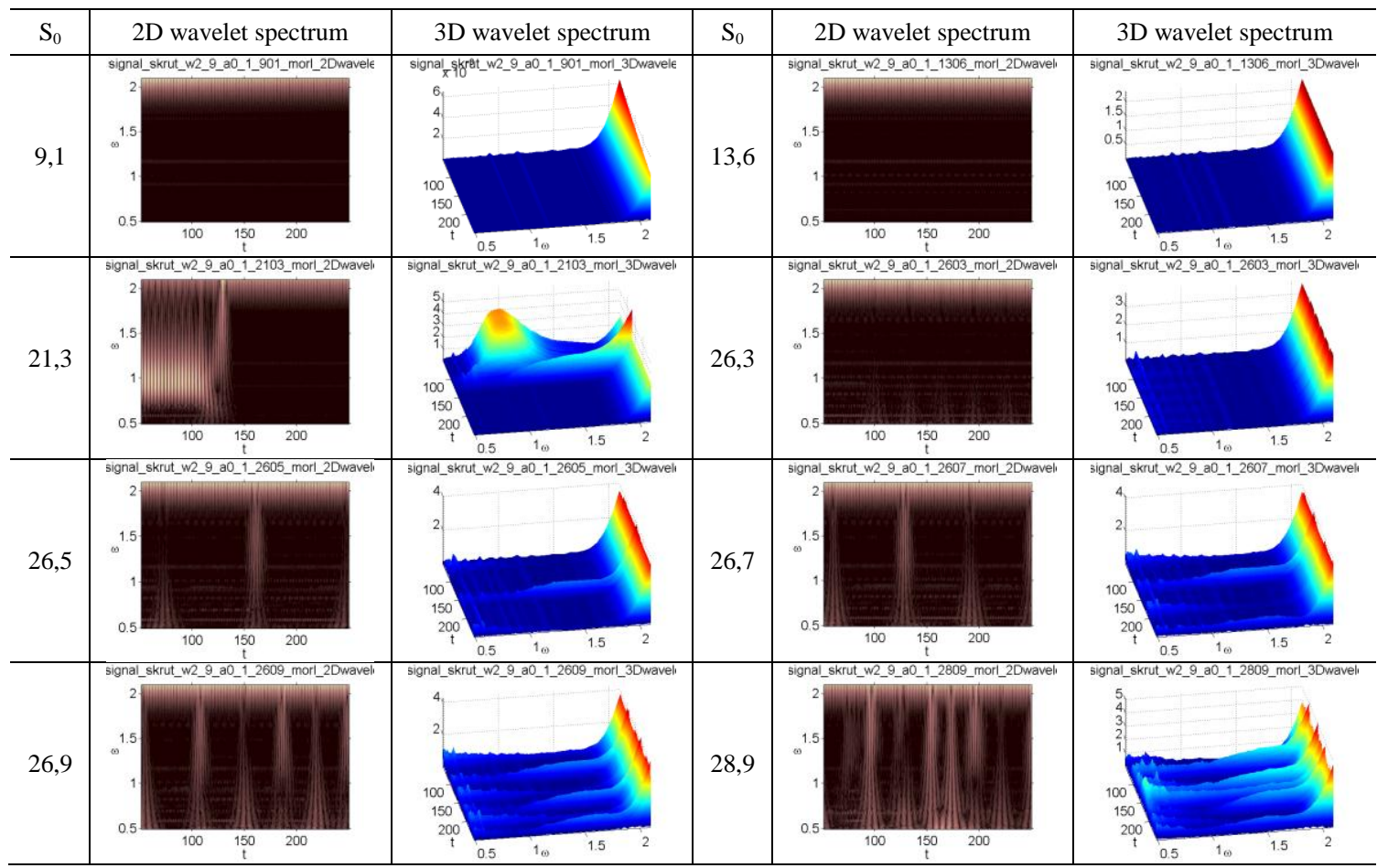

In the wavelet spectrum two linearly independent frequencies appear $\omega_{1}=0.9$ and $\omega_{2}=1.15$, and their power is rather small in comparison to the excitation frequency. This is why the Fourier spectrum exhibits only a periodicity defined by external excitation. Increase of the excitation amplitude awakes a series of frequencies being combinations of $\omega_{1}=0.9, \omega_{2}=1.15$ and $\omega_{\mathrm{p}}=2.9$, and for a long time the plate vibration character is not changed qualitatively. However, for $\mathrm{s}_{0}=21.3$ sudden doubled system re construction takes place in time:

(i) $\mathrm{t}[50 ; 100]$ - here dominate external frequency and $\omega_{1}=0.9$, and the observed vibrations are quasi-periodic with two frequencies;

(ii) $\mathrm{t}[120 ; 140]$ - island of chaotic transitional state appears;

(iii) $\mathrm{t}[150 ; 250]-$ here our plate vibrates with three frequencies $\omega_{\mathrm{p}}=2.9, \omega_{1}=0.9, \omega_{2}=1.15$.

Beginning from $\mathrm{s}_{0}=26.5$ short transitional intermittent and periodic states are observed. Period of intermittency 
window is equal to 30 units, whereas a period between windows of intermittency equals 90 units. Further increase of external compressed loading amplitude causes a decrease of the period between windows of intermittency, and our plate dynamics becomes chaotic.

Since the plate transition from its regular to chaotic dynamics is associated with the occurrence of two irrational frequencies, then the illustrated scenario can be called a modified Ruelle-Takens-Newhouse scenario with intermittency in time.

\section{Conclusions}

Our investigations and analysis indicate that the convergence of numerically obtained results found by FDM can be achieved by monitoring a wavelet spectrum and the Lyapunov exponents. In the case of dynamics in a chaotic zone (contrary to static problems) it is impossible to achieve the convergence of time histories (signals), although one may achieve the integral convergence regarding the wavelet spectrum. In addition, in the case of small external load amplitudes one may achieve convergence with respect to signals, too. An increase of partition numbers in FDM improves the obtained results essentially, but there is a threshold value after which the results cannot be further improved. In this work we have taken $n=14$.

It has been explained and illustrated that frequently used FFT (Fast Fourier Transform) does not make it possible to study a continual system properly and it does not allow us either to detect and monitor transition scenarios into chaos. In other words, we have observed a lack of coincidence of the FFT results with other classical dynamical characteristics. Namely, the obtained FFT indicates the plate harmonic vibration regime, whereas the phase portrait exhibits additional frequencies. It happened because a route to chaos began on frequencies, whose power was so small that it could not be revealed by the FFT.

Furthermore, we have detected and illustrated transition scenarios from periodic to chaotic plate state, where the classical approach based on the Fourier analysis failed. In addition, we have detected and discussed two principally new scenarios. The first one is called the modified Ruelle-Takens-Newhouse scenario with intermittency, i.e. beginning with a certain value of excitation amplitude, short time periodic intervals appear. A further increase of external excitation amplitude causes a decrease of periods between intermittency windows, which finally initiates the occurrence of chaotic plate dynamics. The second scenario is associated with double dynamics reconstruction through subharmonic states before reaching chaos.

\section{Acknowledgment}

J. Awrejcewicz acknowledges support of the Alexander von Humboldt Award.

\section{References}

[1] J. Awrejcewicz and V.A. Krysko, Nonclassic thermo-elastic problem in nonlinear dynamics of shells, Springer - Verlag, Berlin, New York, London, Paris, Tokyo, 2003.

[2] J. Awrejcewicz and V.A. Krysko, Spatial - Temporal chaos and solutions exhibited by Von Karman Model, International Journal of Bifurcations and Chaos 7(12) (2002), 1465-1513.

[3] J. Awrejcewicz and V.A. Krysko, Feigenbaum scenario exhibited by thin plate dynamics, Nonlinear Dynamics 24 (2001), $373-398$.

[4] J. Awrejcewicz and V.A. Krysko, Analysis of complex parametric vibrations of plates and shells using bubnov - Galerkin approach, Archive of Applied Mathematics 73 (2003), 495-504.

[5] J. Awrejcewicz, V.A. Krysko and A.F. Vakakis, Nonlinear dynamics of continuous elastic systems, Springer - Verlag, Berlin, New York, London, Paris, Tokyo, 2004.

[6] Yen-Liang Yeh, Chao's-Kuang Chen and Hsin-Xila, Chaotic and bifurcation dynamics of a simple-supported thermo-elastic circular plate with variable thickness in large deflection, Chaos, Solitons and Fractals 15(5) (2003), 811-829.

[7] R.M. Jones, Buckling of bars, plates and shells, Bull Ridge Publishing, Blacksburg, Virginia, 2006.

[8] Y. Wang, Bifurcation and chaos of bimetallic circular plates subjected to periodic heat load, Z Angew Math Mech 88(4) (2008), 256-266.

[9] Y.X. Hao, L.M. Chen, W. Zhang and I.G. Lei, Nonlinear oscillations, bifurcations and chaos of functionally graded materials plate, Journal of Sound and Vibration 312(4-5) (2008), 862-892. 
[10] W. Zhang, Global and chaotic dynamics for a parametrically excited thin plate, Journal of Sound and Vibration 239(5) (2001), 1013-1036.

[11] Y.X. Sun and S.Y. Zhang, Chaotic dynamics of viscoelastic plates, International Journal of Mechanical Sciences 43(5) (2001), $1195-1208$.

[12] M. Amabili, Nonlinear vibrations and stability of shells and plates, Cambridge University Press, Cambridge, 2008. 

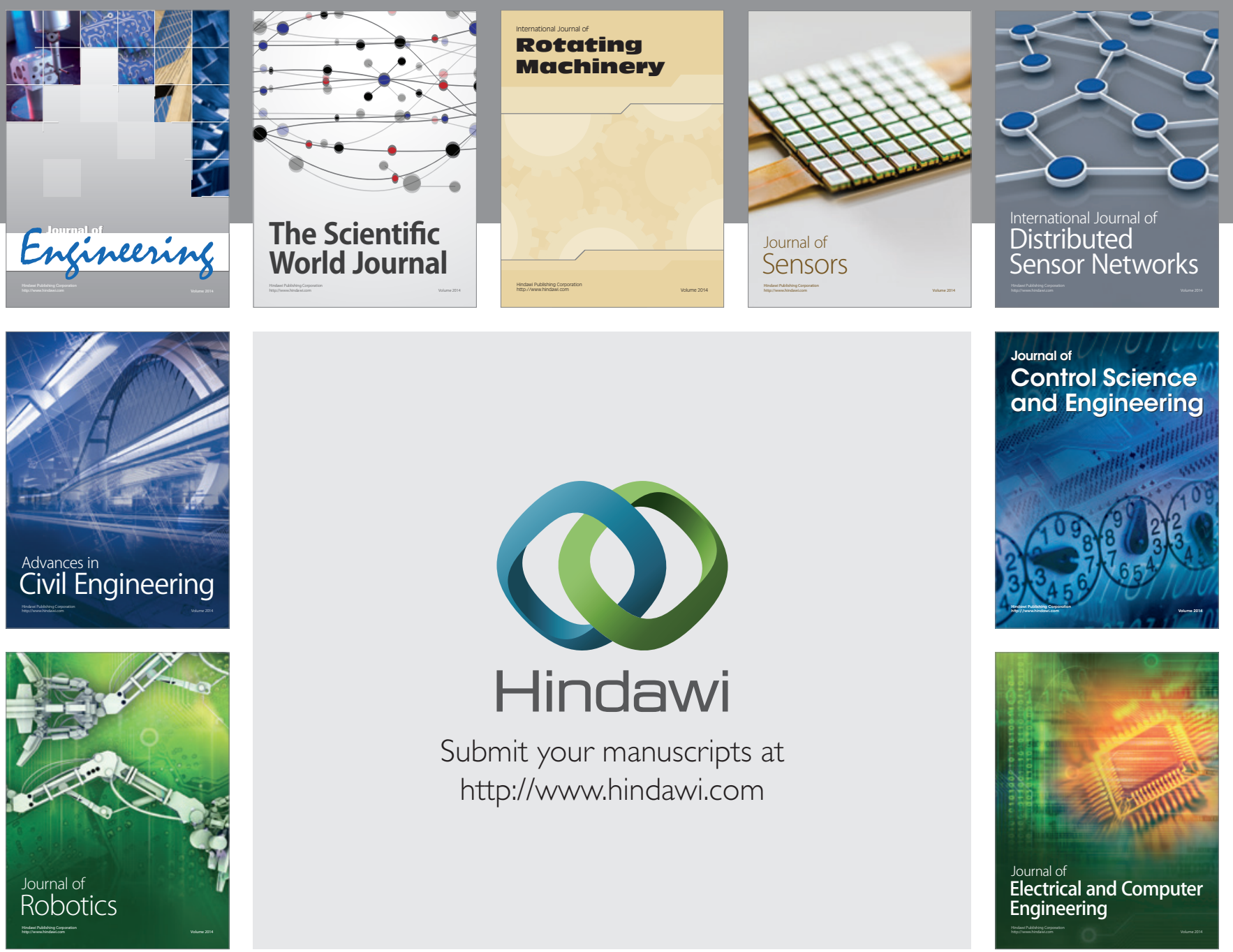

Submit your manuscripts at

http://www.hindawi.com
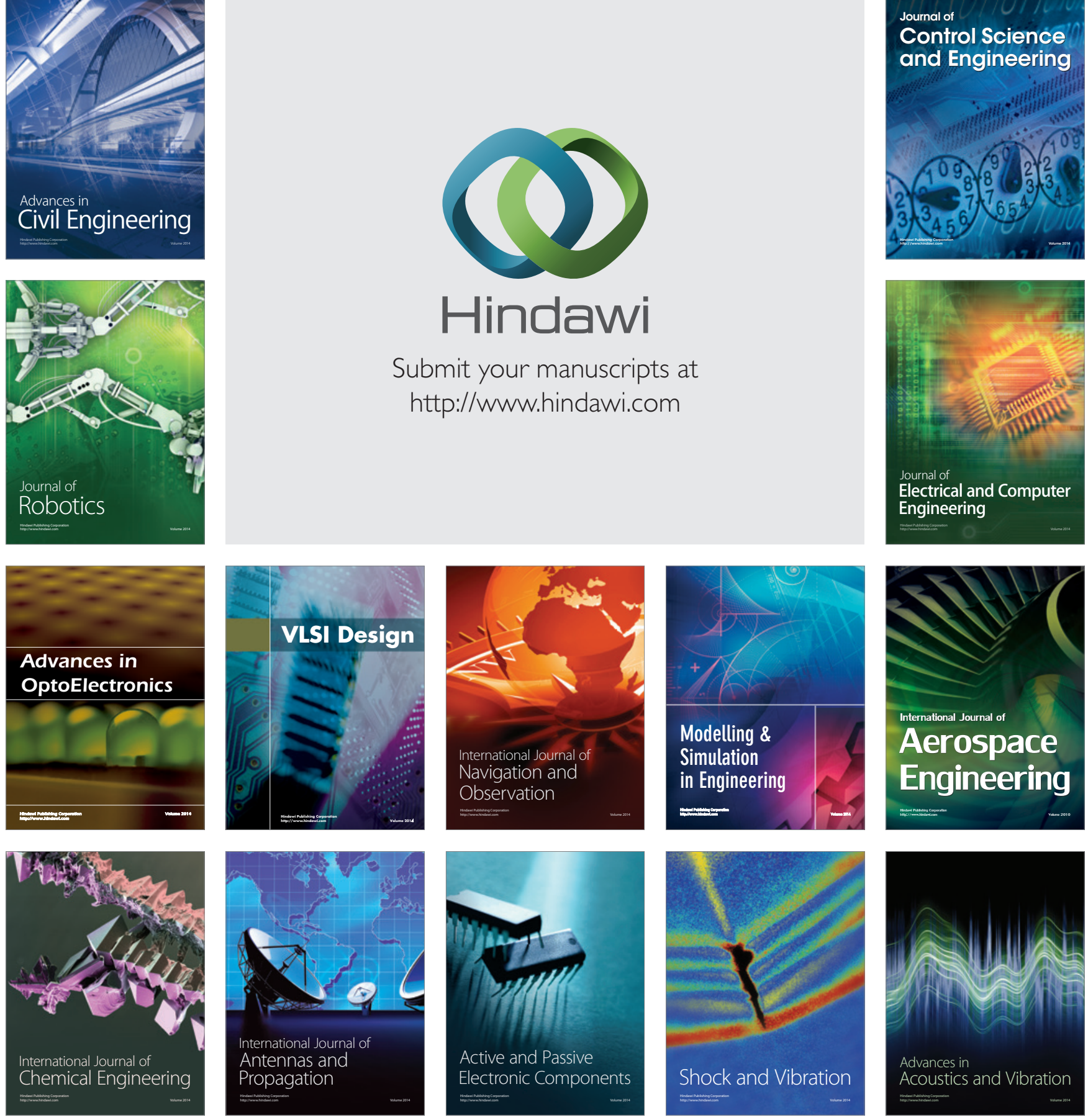